13.1

\title{
Причины низкого значения работы выхода гексаборида лантана как эффективного электронного эмиттера
}

\author{
(c) И.В. Тваури ${ }^{1}$, И.В. Силаев ${ }^{1}$, В.Б. Заалишвили ${ }^{1,2}$, О.Г. Ашхотов ${ }^{1}$, З.Т. Созаев ${ }^{1}$, Т.Т. Магкоев ${ }^{1,2}$ \\ ${ }^{1}$ Северо-Осетинский государственный университет им. К.Л. Хетагурова, Владикавказ, Россия \\ ${ }^{2}$ Геофизический институт - фрилиал Владикавказского научного центра РАН, Владикавказ, Россия \\ E-mail: TT.Magkoev@nosu.ru
}

Поступило в Редакцию 4 июня 2021 г.

В окончательной редакции 19 декабря 2021 г.

Принято к публикации 20 декабря 2021 г.

Для установления механизма высокой эмиссионной эффективности гексаборида лантана как одного из наиболее широко используемых электронных эмиттеров проведено сравнительное исследование состояния атомов La и B в двойной пленочной системе $\mathrm{La}-\mathrm{B}$ с одной стороны и в пленках La и B в отдельности с другой посредством изучения состояния адсорбированных на их поверхности молекул оксида углерода как тестовых частиц, чувствительных к деталям электронного и атомного строения адсорбента. Показано, что формирование дипольного слоя La-B на поверхности является основной причиной высокой эмиссионной эффективности гексаборида лантана. Субнанометровые пленочные системы La-B могут быть перспективны в качестве электронных эмиттеров высокой степени пространственной локальности.

Ключевые слова: тонкопленочные системы, лантан, бор, работа выхода, гексаборид лантана.

DOI: 10.21883/PJTF.2022.06.52202.18903

В последнее время наблюдается вновь возросший интерес к гексабориду лантана вследствие его перспективности в качестве стабильного и высокоэффективного источника электронов, в частности, в наноскопии и нанолитографии [1-3]. Несмотря на повышенный интерес, который привлекает этот материал в течение десятилетий, единой точки зрения на природу присущего ему низкого значения работы выхода не существует. С одной стороны, считается, что низкое значение работы выхода обусловлено электронным строением массивного кристалла $\mathrm{LaB}_{6}$, с другой - формированием поверхностного электроположительного дипольного слоя $\mathrm{La}^{+}-\mathrm{B}^{-}[4,5]$. Как показано ранее, для двойных пленок $\mathrm{La}-\mathrm{B}$ в равновесном состоянии характерна величина работы выхода $(2.6 \mathrm{eV})$, близкая к величине, присущей массивному гексабориду лантана [6]. При этом такая пленочная система обладает довольно высокой стабильностью, что наряду с характерным для нее значением работы выхода позволяет рассматривать ее в качестве прототипа массивного $\mathrm{LaB}_{6}$. Открытым остается вопрос о том, насколько состояние атомов La и B в двойном слое отличается от их состояния в каждом отдельном слое. Вместе с тем именно этот вопрос следует считать ключевым, определяющим синергизм двойного слоя $\mathrm{La}-\mathrm{B}$. B связи с этим целью настоящей работы является установление характера изменения состояния атомов La и В при их совместном осаждении на поверхность подложки $(\mathrm{Mo}(110))$ по сравнению с состоянием в отдельных слоях. Новизна работы заключается в исследовании вопроса о том, насколько состояние атомов $\mathrm{La}$ и $\mathrm{B}$ в двойном слое отличается от их состояния в каждом отдельном слое и какой механизм отвечает за низкое значение работы выхода гексаборида лантана.

Исследования были проведены в условиях сверхвысокого вакуума на установке VGS Escalab MkII (давление остаточных газов $2 \cdot 10^{-10}$ Torr) методами электронной оже-спектроскопии с использованием однокаскадного цилиндрического анализатора с коаксиальной электронной пушкой. Измерения работы выхода осуществлялись методами Андерсона, инфракрасной фурьеспектроскопии и спектроскопии обратного рассеяния ионов низкой энергии с использованием полусферического 150-градусного анализатора Escalab и ионов $\mathrm{He}^{+}$ с энергией $1000 \mathrm{eV}$. Пленки лантана и бора контролируемым образом наносились in situ термическим и электронно-лучевым напылением соответственно. Плотность потока напыляемых атомов и, следовательно, их поверхностная концентрация измерялись с помощью кварцевого резонатора и контролировались измерением ослабления интенсивности оже-сигнала подложки Мо $M N V(188 \mathrm{eV})$ и изменением работы выхода. Адсорбция молекул CO на поверхности пленочных систем La-B, предварительно охлажденных до температуры $95 \mathrm{~K}$, осуществлялась посредством контролируемого напуска газообразного СО в сверхвысоковакуумную камеру до парциального давления $10^{-7}$ Torr. В качестве величины экспозиции СО в $1 \mathrm{~L}$ (Langmuir) принималось значение $10^{-6}$ Torr · s. Для регистрации ИК-спектров использовался инфракрасный фурье-спектрометр Nicolet Nexus 870, адаптированный для измерения колебательных мод адсорбированных молекул в нормальном к плоскости поверхности адсорбента направлении. Для этого ИКлуч, поляризованный в плоскости падения, направлялся 


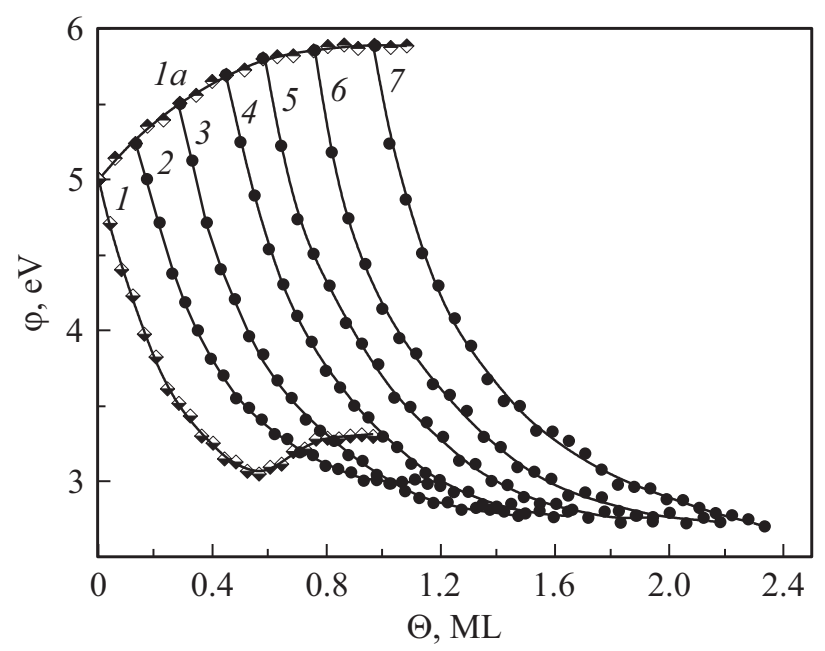

Рис. 1. Зависимость работы выхода от степени покрытия атомов лантана при их адсорбции на поверхности $\mathrm{Mo}(110)$, предварительно покрытой различным количеством атомов боpa. Для удобства все зависимости сгруппированы вместе. Кривые 1 и $1 a-$ соответственно зависимости работы выхода лантана и бора на поверхности Мо(110). Степень покрытия предварительно нанесенных на поверхность Мо(110) атомов бора перед последующим нанесением атомов лантана составляет 0.15 (2), 0.30 (3), 0.50 (4), 0.70 (5), 0.90 (6) и $1.0 \mathrm{ML}(7)$.

на исследуемую поверхность под скользящим углом падения - конфигурация наиболее чувствительная к колебательным свойствам адсорбционных систем рассматриваемого типа [7]. Степень покрытия $(\Theta)$ атомов La и В принималась равной единице $(\Theta=1 \mathrm{ML}$ (монослой)) при их поверхностной концентрации, равной $1.1 \cdot 10^{15}$ и $2.7 \cdot 10^{15} \mathrm{~cm}^{-2}$ соответственно $[6,8]$.

Повышение поверхностной концентрации атомов La на поверхности $\mathrm{Mo}(110)$ при поддержании комнатной температуры приводит к снижению работы выхода до стационарного значения $3.2 \mathrm{eV}$, достигаемого при единичном покрытии, в то время как нанесение атомов бора приводит к повышению работы выхода до значения $5.8 \mathrm{eV}$ (кривые 1, $1 a$ на рис. 1). Нанесение атомов La на поверхность подложки с предварительно нанесенными атомами бора кардинальным образом меняет концентрационную зависимость работы выхода. Одним из возможных объяснений такой существенной трансформации концентрационных зависимостей работы выхода было бы предположение о формировании химических связей между атомами La и B при их совместном осаждении на поверхность подложки. Однако против такого предположения свидетельствует отсутствие химических сдвигов оже-линий La и B в двойной пленке по сравнению с соответствующими одинарными пленками. Двойную пленку $\mathrm{La}-\mathrm{B}$ можно рассматривать как совокупность отдельных атомов La и B без заметных признаков химического связывания. Это приводит к тому, что атомы бора довольно легко диффундируют сквозь пленку лантана на границу с вакуумом даже при комнатной температуре [6]. Такое пространственное перераспределение атомов бора при нанесении атомов лантана можно приписать тому факту, что осажденная на поверхность Мо(110) пленка бора находится, как показано ранее [8], в неравновесном слабо связанном с поверхностью состоянии. Относительно небольшая при этом величина энергии связи бора с поверхностью Мо(110), равная $0.4 \mathrm{eV}$ [8], компенсируется существенно большей величиной энергии адсорбции $\mathrm{La}(4.9 \mathrm{eV})[6]$, индуцирующей отрыв атомов бара с поверхности Мо(110) и их диффузию к границе раздела с вакуумом сквозь пленку лантана.

Для установления того, каково соотношение концентраций атомов лантана и бора в поверхностном слое, непосредственно граничащем с вакуумом, проведены исследования методом спектроскопии обратного рассеяния ионов низкой энергии (СОРИНЭ). Характер изменения спектров в процессе роста концентрации атомов лантана на поверхности пленки бора толщиной $5 \mathrm{ML}$ приведен на рис. 2. По мере роста концентрации атомов La спектральная линия при энергии $327 \mathrm{eV}$, соответствующая рассеянию ионов $\mathrm{He}^{+}$на атомах бора (спектр 1), уменьшается по интенсивности (спектры $2-6)$, но не исчезает даже при значительном покрытии лантана в $30 \mathrm{ML}$ (спектр 6). При этом линия рассеяния при энергии $784 \mathrm{eV}$, соответствующая $\mathrm{La}$, увеличивается по интенсивности, стабилизируясь при покрытии $20 \mathrm{ML}$. При дальнейшем росте покрытия до $30 \mathrm{ML}$ спектр не претерпевает заметных изменений. Это свидетельствует о том, что непосредственно в самом поверхностном слое поддерживается определенное соотношение концентраций атомов лантана и бора. Данное обстоятельство находится в согласии с результатами электронной ожеспектроскопии. Для наглядности соответствующий ожеспектр системы La-B, иллюстрирующий наличие линии бора даже для достаточно толстой пленки лантана поверх слоя бора, приведен на рис. 2 (правая вставка). Для количественной оценки соотношения концентраций атомов La и B проведено сопоставление интегральных интенсивностей линий СОРИНЭ бора для сплошного слоя бора на поверхности $\mathrm{Mo}(110)$ и двойной пленки, образованной нанесением $30 \mathrm{ML} \mathrm{La}$ на пленку бора толщиной $5 \mathrm{ML}$ (левая вставка на рис. 2). С учетом указанной выше величины поверхностной концентрации сплошной пленки бора на поверхности $\mathrm{Mo}(110)$ $\left(2.7 \cdot 10^{15} \mathrm{~cm}^{-2}\right)$ и уменьшения интенсивности приведенных линий СОРИНЭ (линии 1 и 6) в 5.4 раза концентрация атомов бора в самом верхнем слое двойной системы $\mathrm{La}-\mathrm{B}$ составляет $0.5 \cdot 10^{15} \mathrm{~cm}^{-2}$, что приблизительно в 2 раза меньше, чем концентрация атомов лантана $\left(1.1 \cdot 10^{15} \mathrm{~cm}^{-2}\right)$. Это указывает на то, что поверхностный атомный слой двойной пленки $\mathrm{La}-\mathrm{B}$ можно рассматривать как совокупность сопланарно расположенных атомов лантана и бора с соотношением концентраций 2 к 1 соответственно. При этом, несмотря на существенное отличие от соотношения концентраций компонентов в кристалле $\mathrm{LaB}_{6}$ (1 к 6), работа выхода 

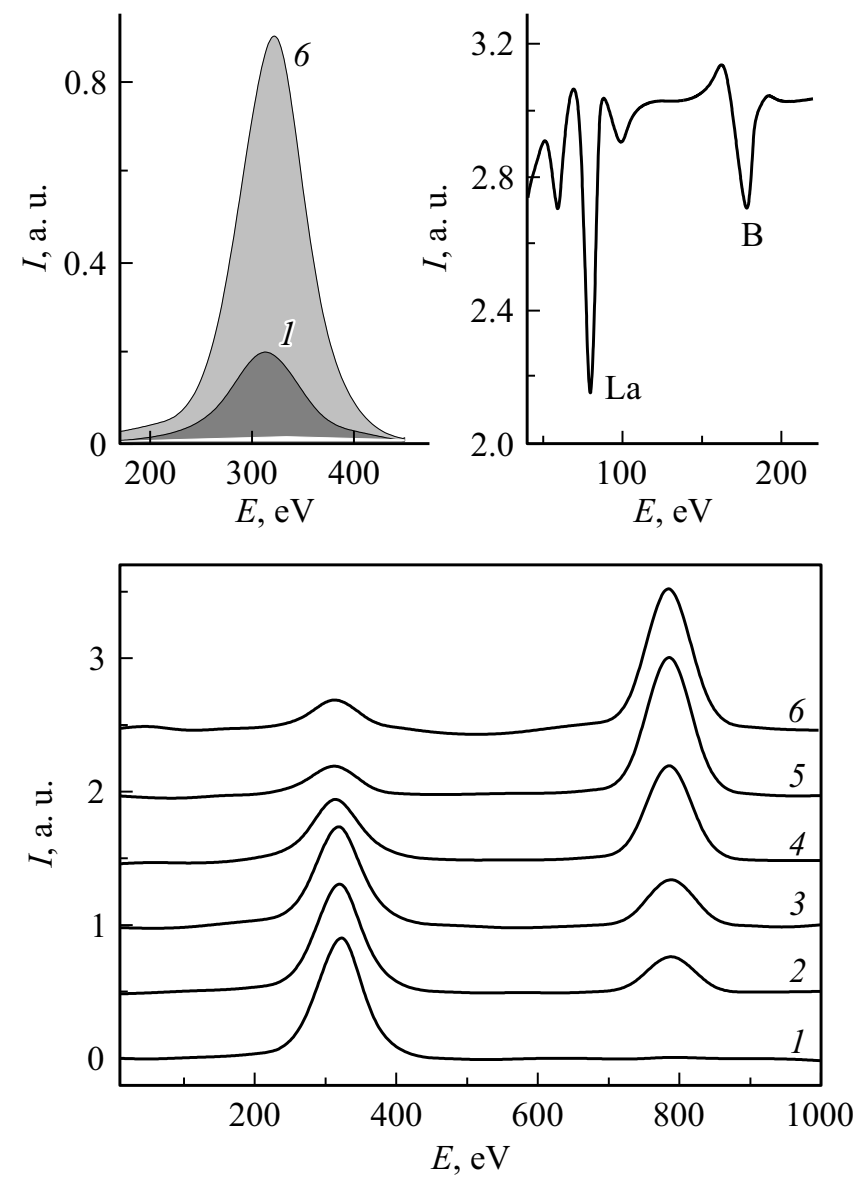

Рис. 2. Характер изменения СОРИНЭ-спектров в процессе роста концентрации атомов лантана на поверхности пленки бора толщиной $5 \mathrm{ML}$. Степень покрытия лантана, ML: $1-0$, $2-5,3-10,4-15,5-20,6-30$. На вставках: справа оже-спектр системы La-B, иллюстрирующий наличие линии бора даже для достаточно толстой пленки лантана поверх слоя бора, слева - сопоставление интегральных интенсивностей линий СОРИНЭ бора для сплошного слоя бора на поверхности $\mathrm{Mo}(110)$ и двойной пленки, образованной нанесением 30 ML La на пленку бора толщиной 5 ML.

пленочной системы тем не менее близка к работе выхода гексаборида лантана.

Для подтверждения данного предположения проведены дополнительные исследования с использованием метода инфракрасной фурье-спектроскопии, направленные на изучение состояния тестовых молекул, а именно $\mathrm{CO}$, адсорбированных на поверхности исследуемых систем. Колебательное состояние таких молекул обладает повышенной чувствительностью к деталям электронного строения подложки [7]. Сопоставление ИКспектров молекул $\mathrm{CO}$, адсорбированных на отдельных пленках La и $\mathrm{B}$ толщиной $3 \mathrm{ML}$ и на равновесной двойной пленке $\mathrm{La}-\mathrm{B}$, охлажденных до температуры $95 \mathrm{~K}$, приведены на рис. 3. Спектры, соответствующие отдельным пленкам, состоят из линий при значениях волнового числа 2082 и $2160 \mathrm{~cm}^{-1}$ для La и B соот- ветственно (спектры 1 и 3). Эти значения волновых чисел соответствуют внутримолекулярным колебаниям $\mathrm{CO}$, связанным по линейной геометрии непосредственно с атомами La и В. ИК-спектр, соответствующий $\mathrm{CO}$ на поверхности двойной системы La-B (спектр 2), с достаточно хорошей точностью может быть представлен в виде суперпозиции линий $\mathrm{CO}$ на отдельных пленках $\mathrm{La}$ и В. При этом наблюдается довольно незначительный фиолетовый сдвиг $\left(8 \mathrm{~cm}^{-1}\right)$ компоненты, соответствующей $\mathrm{La}$, в то время как энергетическое положение компоненты для В не претерпевает заметных изменений по сравнению со случаем отдельных пленок. Такое подобие ИК-спектров для отдельных пленок La и В и двойной пленочной системы $\mathrm{La}-\mathrm{B}$ подтверждает предположение об отсутствии химического взаимодействия между атомами La и B.

В результате проведенного исследования можно сделать вывод, что в равновесном состоянии двойная тонкопленочная система $\mathrm{La}-\mathrm{B}$ толщиной от единиц до долей нанометра на поверхности $\mathrm{Mo}(110)$ имеет величину работы выхода, близкую к таковой для массивного гексаборида лантана. При этом не обнаружено формирования химической связи между атомами лантана и бора. Последние имеют состояние, близкое к наблюдаемому для отдельных пленок La и B. Низкое значение работы выхода в пленочной системе $\mathrm{La}-\mathrm{B}$ следует считать обусловленным формированием электроположительного слоя в приповерхностной области системы за счет поляризации заряда $\mathrm{La} \rightarrow \mathrm{B}$. Близость значений работы выхода двойной пленочной системы $\mathrm{La}-\mathrm{B}$ и массивного гексаборида лантана свидетельствует в пользу

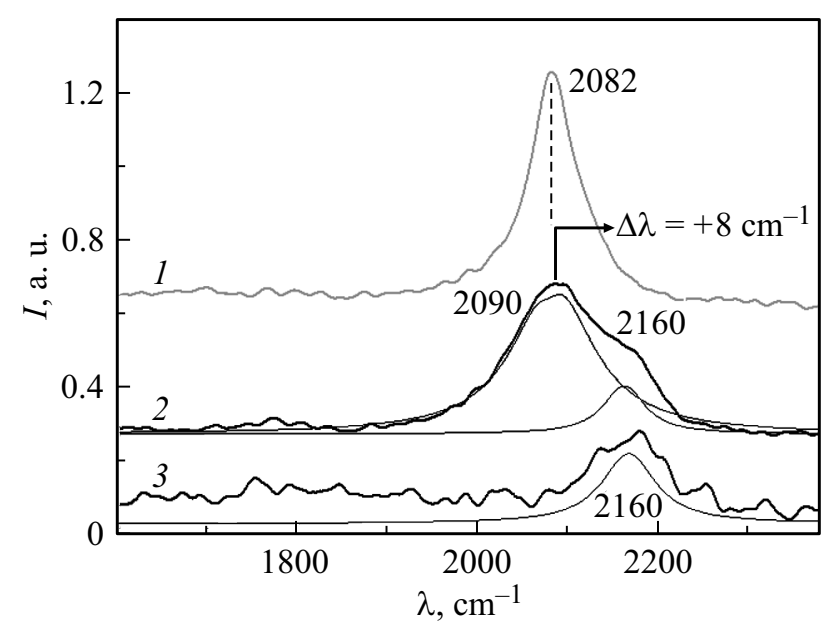

Рис. 3. ИК-спектры молекул СО, адсорбированных на поверхности отдельных пленок лантана (1) и бора (3) толщиной $3 \mathrm{ML}$ и на поверхности двойной пленки $\mathrm{La}(2 \mathrm{ML})-\mathrm{B}$ (2 ML) (2). Температура подложки $95 \mathrm{~K}$. Для наглядности демонстрации компонент спектров 2 и 3 приведена их аппроксимация функциями Гаусса с максимумами при значениях волновых чисел 2090 и $2160 \mathrm{~cm}^{-1}$ (спектр 2) и $2160 \mathrm{~cm}^{-1}$ (спектр 3). Волновое число максимума спектра $2\left(2090 \mathrm{~cm}^{-1}\right)$ смещено в фиолетовую область относительно максимума спектра 1 $\left(2082 \mathrm{~cm}^{-1}\right)$ на $8 \mathrm{~cm}^{-1}\left(\Delta \lambda=+8 \mathrm{~cm}^{-1}\right)$. 
того, что высокая эмиссионная эффективность последнего обусловлена не столько особенностями его электронного строения, сколько формированием дипольного слоя $\mathrm{La}^{+}-\mathrm{B}^{-}$на его поверхности. С прикладной точки зрения возможность замены массивного электронного источника на основе $\mathrm{LaB}_{6}$ соответствующей наноразмерной пленочной системой имеет перспективу применения в наноскопии и нанолитографии, в частности, для дальнейшего уменьшения размеров исследуемых и формируемых систем.

\section{Финансирование работы}

Работа выполнена на кафедре физики конденсированного состояния Северо-Осетинского государственного университета с использованием оборудования лабораторий физики адсорбционных явлений и физики поверхности и катализа в рамках госзадания Минобрнауки РФ (код научной темы FEFN-2021-0005).

\section{Конфликт интересов}

Авторы заявляют, что у них нет конфликта интересов.

\section{Список литературы}

[1] G.-C. Potrivitu, L. Xu, S. Huang, M.W.A.B. Rohaizat, S. Xu, J. Appl. Phys., 127, 64501 (2020). DOI: 10.1063/1.5142019

[2] C. Uechi, K. Ohoyama, Y. Fukumoto, Y. Kanazawa, N. Happo, M. Harada, Y. Inamura, K. Oikawa, W. Matsuhra, F. Iga, A.K.R. Ang, K. Hayashi, Phys. Rev. B, 102, 054104 (2020). DOI: 10.1103/PhysRevB.102.054104.

[3] G. Singh, R. Bücker, G. Kassier, M. Barthelmess, F. Zheng, V. Migunov, M. Kruth, Appl. Phys. Lett., 113, 093101 (2018). DOI: $10.1063 / 1.5039441$

[4] H. Kawano, Prog. Surf. Sci., 83, 1 (2008). DOI: 10.1016/j.progsurf.2007.11.001

[5] M. Bakr, M. Kawai, T. Kii, H. Zen, K. Masuda, H. Ohgaki, J. Appl. Phys., 117, 064503 (2015). DOI: 10.1063/1.4908004

[6] T.T. Magkoev, G.G. Vladimirov, G.A. Roump, Surf. Sci., 602, 1705 (2008). DOI: 10.1016/j.susc.2008.03.002

[7] C. Hirschmugl, Surf. Sci., 500, 577 (2002). DOI: $10.1016 / \mathrm{S} 0039-6028(01) 01523-0$

[8] T.T. Magkoev, A.M. Turiev, N.I. Tsidaeva, D.G. Panteleev, G.G. Vladimirov, G.A. Rump, J. Phys.: Condens. Matter, 20, 485007 (2008). DOI: 10.1088/0953-8984/20/48/485007 